\title{
ERK1/2-Egr-1 Signaling Pathway-Mediated Protective Effects of Electroacupuncture in a Mouse Model of Myocardial Ischemia-Reperfusion
}

\author{
Juan Zhang, ${ }^{1}$ Jiangang Song, ${ }^{1}$ Jin $\mathrm{Xu},{ }^{1}$ Xuemei Chen, ${ }^{1}$ \\ Peihao Yin, ${ }^{2}$ Xin $\mathrm{Lv}^{3}{ }^{3}$ and Xiangrui Wang ${ }^{1}$ \\ ${ }^{1}$ Department of Anesthesiology, Renji Hospital, School of Medicine, Shanghai Jiao Tong University, 1630 Dongfang Road, \\ Shanghai 200127, China \\ ${ }^{2}$ Department of General Surgery, Putuo Hospital, Shanghai University of Traditional Chinese Medicine, Shanghai 200062, China \\ ${ }^{3}$ Department of Anesthesiology, Shanghai Pneumology Hospital, School of Medicine, Tongji University, Shanghai 200433, China
}

Correspondence should be addressed to Xiangrui Wang; wangxiangrui2010@gmail.com

Received 12 February 2014; Accepted 10 April 2014; Published 5 May 2014

Academic Editor: Sergio-Botelho Guimarães

Copyright (C) 2014 Juan Zhang et al. This is an open access article distributed under the Creative Commons Attribution License, which permits unrestricted use, distribution, and reproduction in any medium, provided the original work is properly cited.

\begin{abstract}
Early growth response- (Egr-) 1 is an upstream master switch in controlling inflammatory responses following myocardial ischemiareperfusion (I/R). Activation of extracellular signal-regulated protein kinase-1 and kinase-2 (ERK1/2) signaling is known to upregulate Egr-1. ERK1/2 pathway has been previously shown to mediate the therapeutic action of electroacupucture (EA). Thus, we hypothesized that EA would reduce myocardial I/R injury and inflammatory responses through inhibiting Egr-1 expression via the ERK1/2 pathway. Mice were pretreated with EA, U0126, or combination of EA and U0126 and then underwent $1 \mathrm{~h}$ myocardial ischemia and $3 \mathrm{~h}$ reperfusion. We investigated that EA significantly attenuated the I/R-induced upregulation of both Egr- 1 and phosporylated-ERK1/2 (p-ERK1/2), decreased myocardial inflammatory cytokines including tumor necrosis factor- $\alpha$ (TNF- $\alpha$ ) and interleukin-1 $\beta$ (IL-1 $\beta$ ), and reduced the infarct size and the release of cardiac troponin I (cTnI). U0126 treatment also exhibited the same effect as EA on Egr-1 level and subsequent cardioprotective effects. There was no additive effect of cotreatment with EA and U0126 on the expression of Egr-1 and its downstream target genes (TNF- $\alpha$, IL-1 $\beta$ ) or serum cTnI level. Collectively, these observations suggested that EA attenuates myocardial I/R injury, possibly through inhibiting the ERK1/2-Egr-1 signaling pathway and reducing the release of proinflammatory cytokines.
\end{abstract}

\section{Introduction}

Acupuncture is a therapeutic technique that originated in China more than five thousand years ago [1]. Comparing with traditional manual acupuncture, electroacupuncture (EA) is more repeatable and adjustable. Accumulating evidences from experimental studies indicated that EA at selected acupoints [e.g., Neiguan (PC6)] can reduce myocardial ischemia-reperfusion $(\mathrm{I} / \mathrm{R})$ injury, as reflected by reducing release of myocardial enzyme such as cTnI and creatine phosphokinase (CPK) $[2,3]$, attenuating the frequency and severity of arrhythmias $[4,5]$ and decreasing infarct size [68]. More importantly, the beneficial effects of EA have also been observed in clinical settings, where it has resulted in reduced cTnI release, decreased C-reactive protein level, and shorter intensive care unit stay in both adult and pediatric patients receiving heart surgeries $[2,9]$. Despite these visible benefits of EA, the underlying molecular mechanisms of EAmediated cardiac protection remain unclear.

Early growth response- (Egr-) 1, a transcription factor, has been shown to be upregulated in the heart $[10,11]$ and initiate inflammation following I/R [12]. Using an Egr1 antisense oligodeoxyribonucleotide or a catalytic deoxyribonucleic acid molecule (DNAzyme) to inhibit Egr-1 has been shown to reduce myocardial inflammation and protect heart function against the I/R [11, 13]. Studies using other disease models have also pointed to the regulation of Egr1 by the ERK1/2 pathway. In lung I/R, Egr-1 requires the 
activation of ERK1/2 to exert its proinflammatory effects [14]. Upregulation of Egr-1 via the ERK1/2 pathway also contributes to the damage to pulmonary artery smooth muscle cells in a model of chronic hypoxia [15]. Recently, the ERK1/2 pathway has been implicated in the therapeutic effects of EA. It is reported that EA at PC6 acupoints alleviates cardiac hypertrophy after myocardial infarction by inhibiting the activation of ERK1/2 pathway [16]. Collectively, it suggests a link among EA, Egr-1, and ERK1/2 signaling pathway. Thus, we tested the hypothesis that EA attenuates myocardial I/R injury by inhibiting the ERK1/2-Egr-1 pathway.

\section{Materials and Methods}

2.1. Ethics Statements. All experimental protocols were approved by Animal Care Committee of Shanghai Jiao Tong University, Shanghai, China. All experiments conformed to the Guide for the Care and Use of Laboratory Animals published by the National Institutes of Health (NIH Publication, 8th Edition, 2011). For the experiments described here, we used a total of 153 male C57BL6 mice (8-10 weeks of age) from Sino-British SIPPR/BK Lab Animals (Shanghai, China). Animals were maintained under a $12 / 12 \mathrm{~h}$ light/dark cycle at $24^{\circ} \mathrm{C} \pm 1^{\circ} \mathrm{C}$, with unrestricted access to standard food and water.

2.2. Experiment Design. The hypothesis schematic diagram and study protocol diagram are depicted in Figures S1 and S2 in Supplementary Material available online at http://dx.doi.org/10.1155/2014/253075. Three sets of experiments were conducted to investigate whether the ERK1/2Egr-1 pathway is involved in the protective effects of EA against myocardial I/R injury. In experiment 1 , thirty mice were used to examine the temporal profile of Egr-1 and pERK1/2 after $1 \mathrm{~h}$ ischemia followed by reperfusion of varying lengths of time $(0,3,6$, and $24 \mathrm{~h} ; n=6$ for each time point). Based on the maximal expression of both Egr-1 and ERK1/2 observed in this experiment, reperfusion duration was set at $3 \mathrm{~h}$ for subsequent experiments (except for infarct size determination). Then we evaluated the effects of EA on Egr1 and ERK1/2 expression during myocardial I/R, and mice were exposed to sham surgery (SHAM), I/R alone (IR), or EA prior to I/R (EA + IR, EA was performed $30 \mathrm{~min}$ prior to the surgery and lasted until the start of surgery) ( $n=$ 12 /group). In experiment 2, the effects of U0126 [a highly selective inhibitor of ERK kinase; Cell Signaling Technology, Danvers, USA; $20 \mathrm{mg} / \mathrm{kg}, 1 \mathrm{~h}$ prior to the surgery, i.p. [17]] were compared to vehicle treatment $(0.1 \% \mathrm{v} / \mathrm{v}$ DMSO $)$ as well as I/R alone ( $n=12$ /group). In experiment 3 , mice received EA, U0126, or both treatments prior to the surgery ( $n=9$ /group). Areas at risk (AAR) from the left ventricles (LV) were collected to measure the content of Egr-1, pERK1/2, and ERK1/2 using western blot, real-time PCR, and immunohistochemical staining. Myocardial levels of TNF- $\alpha$ and IL- $1 \beta$ were also measured. Serum was collected for the cTnI assay. Six mice per group were used to determine infarct size at $24 \mathrm{~h}$ after reperfusion.
2.3. Myocardial I/R Injury. Mice were anesthetized with $2 \%$ isoflurane (in 100\% oxygen) under artificial ventilation using a rodent ventilator (Kent Scientific Co., Torrington, Connecticut, USA). The adequacy of anesthesia was verified using tail pinch. The heart was exposed at the fourth intercostal space, and the left anterior descending coronary artery (LAD) was occluded transiently using a 6-0 suture and reperfused for varying duration as described previously [18]. The incision was closed after the procedure, and the mice were allowed to recover from the anesthesia. Before the mice were sacrificed, AAR from the LV and blood from the inferior vena cava were collected for biochemical analyses. The body temperature was maintained at $37^{\circ} \mathrm{C}$ throughout the study.

2.4. Electroacupuncture. EA was delivered to PC6 acupoints bilaterally, at $1 \mathrm{~mm}$ above the wrist joint between the radius and ulna on the ventral surface of the forelimb [19]. Briefly, stainless needles were inserted to a depth of $3 \mathrm{~mm}$ and secured using plastic adhesive tape. Electrical stimulation (current of $1 \mathrm{~mA}$, alternating dense and disperse mode, $2 \mathrm{~Hz}$ [0.6-ms pulse width] versus $100 \mathrm{~Hz}$ stimulation [0.2-ms pulse width], each lasting for $3 \mathrm{~s}$ ) was delivered using an electrical stimulation device (HANS LH-202, Huawei Co., Beijing, China) for $30 \mathrm{~min}$ [20].

2.5. Determination of Infarct Size. Infarct size was evaluated by Evans blue and triphenyltetrazolium chloride (TTC) (Sigma-Aldrich Co., St. Louis, MO, USA) staining as described previously [21]. Briefly, LAD was religated at $24 \mathrm{~h}$ after the reperfusion followed by injection of $2 \%$ Evans blue into the aortic arch. The heart was sliced transversely into five blocks of equal thickness, incubated in 1\% TTC for $15 \mathrm{~min}$ at $37^{\circ} \mathrm{C}$, and fixed in $10 \%$ formalin overnight. Images were digitally captured using a microscope (DFC500, LEICA, Solms, Germany) and a digital camera (C-DSD230, Nikon, Tokyo, Japan). The LV area, AAR and infarct area (IA) were determined with planimetry software (Image J; National Institute of Health, Bethesda, USA) and adjusted for the weight. The ratio of AAR to LV area and IA to AAR was calculated.

2.6. Western Blot Analysis. The LV was frozen in liquid nitrogen and stored at $-80^{\circ} \mathrm{C}$ until homogenization on ice using an EDTA-free buffer containing protease inhibitor cocktail (Roche Ltd., Basel, Switzerland). The homogenate was centrifuged for $15 \mathrm{~min}$ at $12,000 \mathrm{rpm}$ at $4^{\circ} \mathrm{C}$. Protein concentration of the supernatant was determined using a BCA kit (Thermo Scientific, Middletown, USA). Equal amounts of protein $(40 \mu \mathrm{g})$ were fractionated in $12 \%$ SDS-polyacrylamide gels and transferred onto nitrocellulose membranes. The membranes were blocked with 5\% nonfat dry milk and 0.01\% Tween-20 in Tris-buffered saline (TBS) at $\mathrm{pH} 7.6$ prior to incubation with monoclonal antibodies against Egr1, p-ERK1/2, ERK1/2 (Cell Signaling Technology, Danvers, USA), or GAPDH (Kangcheng, Shanghai, China) at $4^{\circ} \mathrm{C}$ overnight. After incubation with an anti-rabbit secondary antibody (Biotime, Shanghai, China), the bands were visualized and analyzed using a BIO-RAD system (Molecular 
Imager ChemiDoc XPS+, Hercules, USA). GAPDH was used as an internal control.

2.7. Quantitative Real-Time Polymerase Chain Reaction. Egr$1 \mathrm{mRNA}$ in the LV was measured using quantitative real-time polymerase chain reaction (qRT-PCR) with SYBER Premix Ex Taq and Primescript RT reagent Kit (Takara, Otsu, Japan) and expressed as $2^{-\Delta \Delta \mathrm{Ct}}$ (relative fold change). GAPDH was used as an internal control. The primers for Egr-1 were forward $5^{\prime}$-GCCTTAAGGGGGTAGGAGTG- $3^{\prime}$ and reverse $5^{\prime}$-CCTCTTCCTCATCGTGCTCT- $3^{\prime}$. The primers for GAPDH were forward $5^{\prime}$-GGTTGTCTCCTGCGACTTC- $3^{\prime}$ and reverse $5^{\prime}$-CCTGTTGCTGTAGCCGTATTCAT- ${ }^{\prime}$. The PCR was conducted using a standard cycle: $95^{\circ} \mathrm{C}$ for 10 minutes, followed by 40 cycles of $95^{\circ} \mathrm{C}$ for 15 seconds, $56^{\circ} \mathrm{C}$ for 30 seconds, and $72^{\circ} \mathrm{C}$ for 30 seconds.

2.8. Immunohistochemical Staining. The LV was fixed in $4 \%$ paraformaldehyde and embedded in paraffin for sectioning into $4 \mu \mathrm{m}$ sections. After antigen retrieval, the sections were incubated with a primary antibody against Egr-1 followed by incubation with a biotin-conjugated secondary antibody and color reaction with avidin-peroxidase (ABC kit) and a $\mathrm{DAB}$ substrate kit (Vector Laboratories, Burlingame, USA). The sections were counterstained with hematoxylin. Images were digitized using a microscope (BX-51; Olympus, Tokyo, Japan) and analyzed using Image $J$ software. The measurement was performed by two researchers blinded to the treatment condition.

2.9. ELISA. TNF- $\alpha$ and IL- $1 \beta$ in myocardial homogenate were examined using ELISA reagent kits (R\&D systems, Minneapolis, USA). Serum cTnI was determined using an ELISA kit (Life Diagnostics, West Chester, USA).

2.10. Statistical Analysis. Data are expressed as the mean \pm SEM and statistically analyzed by the Statistical Package for the Social Sciences (SPSS, Version 16.0; SPSS Inc., Chicago, USA). Independent Student's $t$-test was used when comparing the infarct size between IR and EA + IR group. All the other data were analyzed with one-way ANOVA followed by Turkey's post-test. $P<0.05$ was considered statistically significant.

\section{Results}

3.1. EA Pretreatment Inhibited Myocardial Egr-1 and p-ERK1/2 Expression, Decreased Inflammatory Cytokines, and Reduced Infarct Size. Varying reperfusion time points demonstrated changes in mRNA and protein levels of Egr-1 paralleled that of p-ERK1/2. Nearly all time points exhibiting significant increases from Sham controls and peak levels were observed after $3 \mathrm{~h}$ of reperfusion $(P<0.001$ versus SHAM; Figures $1(\mathrm{a})-1(\mathrm{~d}))$. Using $3 \mathrm{~h}$ of reperfusion, we tested the effects of EA on myocardial Egr-1 and p-ERK1/2 levels. EA treatment significantly attenuated the I/R-induced increase in Egr-1 $(P=$ 0.036 for protein, $P<0.001$ for mRNA; EA + IR versus IR) and $\mathrm{p}$-ERK1/2 $(P=0.030$ for $\mathrm{p}$-ERK1, $P=0.037$ for $\mathrm{p}$-ERK2;
EA + IR versus IR; Figures 2(a)-2(d)). Immunohistochemical staining also revealed that EA attenuated Egr-1 expression in response to I/R (Figures 2(e)-2(f)). In addition, the infarct size (IA/AAR) is significantly smaller in EA pretreatment group than the I/R alone group ( $28.15 \pm 1.59 \%$ versus $42.64 \pm$ $2.83 \%, P=0.019$; Figures 3(a)-3(b)). EA decreased the cTnI release in the serum $(23.16 \pm 1.25$ versus $13.03 \pm 1.89 \mathrm{ng} / \mathrm{mL}$ for I/R controls, $P=0.006$; Figure 3(c)) and led to a reduction in myocardial TNF- $\alpha(14.66 \pm 1.67$ versus $9.78 \pm 1.19 \mathrm{pg} / \mathrm{mL}$ for I/R controls, $P=0.043$, Figure $3(\mathrm{~d}))$ and IL-1 $\beta$ levels (18.62 \pm 1.95 versus $12.97 \pm 1.18 \mathrm{pg} / \mathrm{mL}$ for $\mathrm{I} / \mathrm{R}$ controls, $P=0.035$, Figure 3(e)). These findings indicated a protective effect of EA against the myocardial damage and inflammatory injury.

\subsection{Activation of the ERK1/2 Pathway Is Responsible for Egr-1} Upregulation and Myocardial Injury following $I / R$. In order to determine the effects of ERK1/2 inhibition on I/R injury, we used U0126 to block the activation of ERK1/2 in animals undergoing I/R surgery. U0126 treatment significantly inhibited p-ERK1/2 $(P=0.003$, U0126 + IR versus DMSO + IR; Figures 4(a) and 4(c)). Additionally, U0126 treatment significantly decreased both Egr-1 protein $(P=0.026)$ and mRNA levels $(P<0.001$, U0126 + IR versus DMSO + IR; Figures 4(a), 4(b), and 4(d)). Immunohistochemical analysis revealed fewer myocardial Egr-1-positive cells in mice receiving U0126 in comparison to the vehicle control (Figures 4(e)-4(f)). In addition, the infarct size (IA/AAR) in the U0126 + IR group was significantly reduced when compared with the DMSO + IR group $(P=0.029,23.62 \pm$ $1.43 \%$ versus $41.03 \pm 2.03 \%$; Figures $5(\mathrm{a})-5(\mathrm{~b}))$ as well as the serum cTnI levels $(P=0.034,23.24 \pm 0.74$ versus $17.67 \pm$ $2.33 \mathrm{ng} / \mathrm{mL}$; Figure 5(c)). The myocardial TNF- $\alpha$ and IL- $1 \beta$ levels in U0126 + IR group were attenuated when compared with DMSO + IR group $(P=0.035$ for TNF- $\alpha$ : $13.66 \pm 1.56$ versus $9.65 \pm 0.94 \mathrm{pg} / \mathrm{mL} ; P=0.043$ for IL-1 $\beta$ : $17.62 \pm 1.59$ versus $12.97 \pm 0.54 \mathrm{pg} / \mathrm{mL}$; Figures 5(d) $-5(\mathrm{e}))$.

\subsection{Combination of EA and ERK1/2 Inhibition Did Not Further} Enhance Cardiac Protection against I/R Injury. As shown previously, both EA and U0126 treatment had favorable effects on myocardial damage and inflammation processes when given separately. However, effects of combined treatment of EA and U0126 did not differ from either EA or U0126 alone when comparing myocardial Egr-1 and p-ERK1/2 expression $(P>$ 0.05; Figures 6(a)-6(c)). Additionally, EA + U0126 did not further affect the myocardial level of TNF- $\alpha$, IL- $1 \beta$, or serum cTnI levels in comparison to EA or U0126 alone $(P>0.05$; Figures 6(d)-6(f)).

\section{Discussion}

The current study confirmed the cardiac protection of EA against $\mathrm{I} / \mathrm{R}$ reflected by reduced infarct size and lower serum cTnI level, which is consistent with work done by our group and others $[3,22,23]$. Moreover, we demonstrated that EA at PC6 acupoints significantly attenuated I/R-induced upregulation of Egr-1 as well as ERK1/2 activation in the myocardium. Additionally, blocking ERK1/2 activation with 


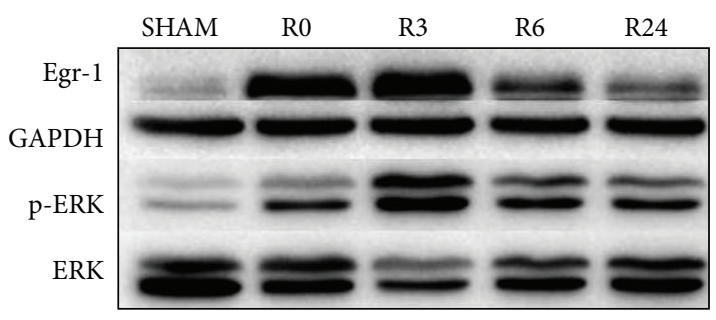

(a)

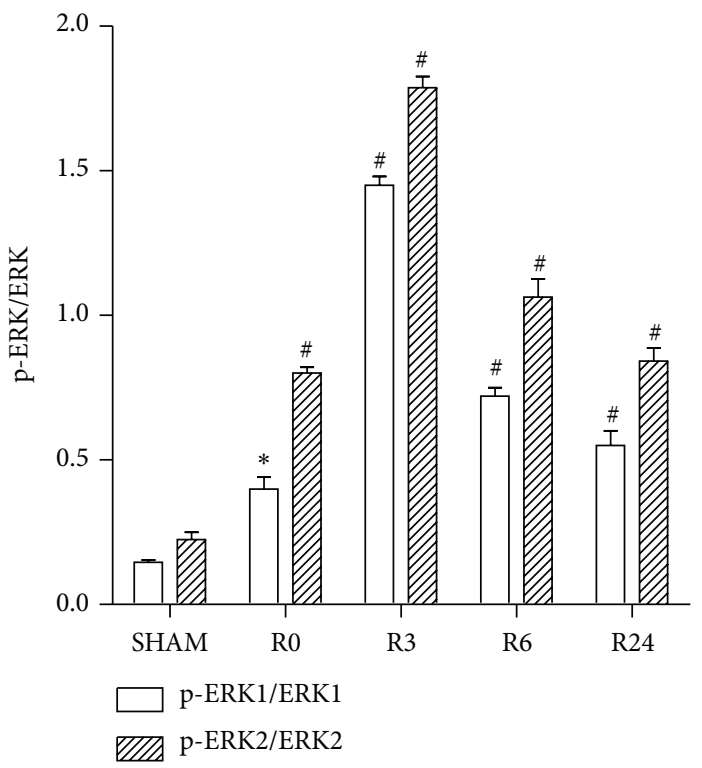

(c)

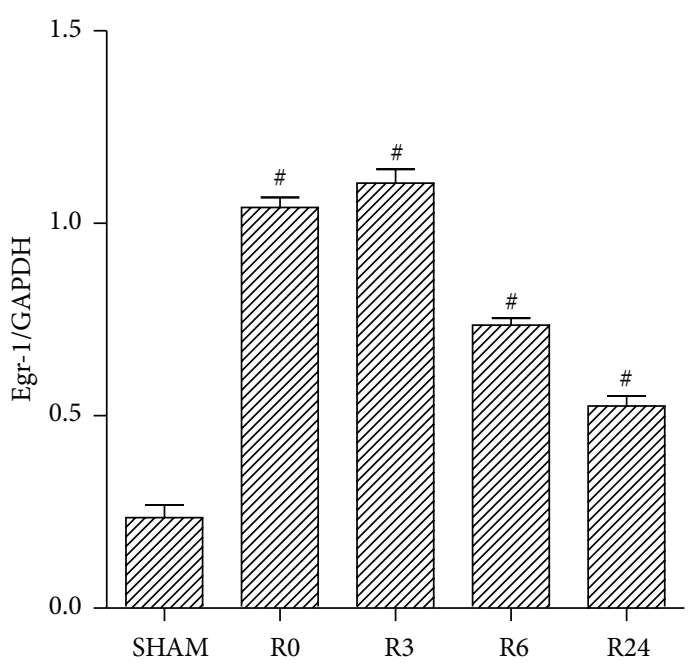

(b)

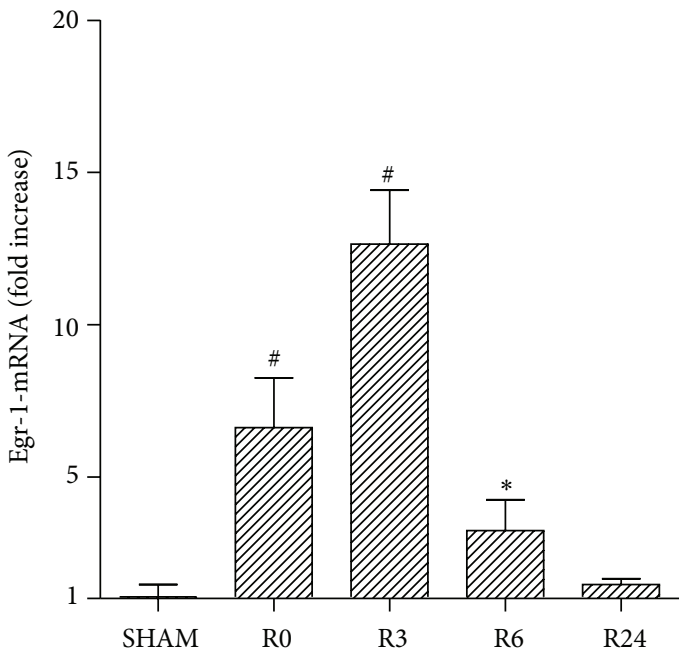

(d)

FIGURE 1: Comparison of the myocardial Egr-land p-ERK1/2 expression at different time points after I/R. The protein levels of Egr-1and pERK1/2 at varying reperfusion time points (R0, R3, R6, and R24) were determined by western blot (a) and the corresponding densitometric analysis is shown in (b, c). Besides, the mRNA levels of Egr-1 were measured by qRT-PCR with data presented as relative fold increase versus sham control (d). For Egr-1 and p-ERK1/2, nearly all time points exhibit significant increase comparing with sham controls and peak levels are observed at R3 time point $(P<0.001$, R3 versus SHAM). Six mice were sacrificed at each time points for protein and mRNA determination. R0 $=$ ischemia for $1 \mathrm{~h} ; \mathrm{R} 3=$ ischemia for $1 \mathrm{~h}+$ reperfusion for $3 \mathrm{~h}$; 6 = ischemia for $1 \mathrm{~h}+$ reperfusion for $6 \mathrm{~h}$; $\mathrm{R} 24=$ ischemia for $1 \mathrm{~h}+$ reperfusion for $24 \mathrm{~h}$. Data are presented as mean $\pm \mathrm{SEM} ;{ }^{*} P<0.05$ and ${ }^{*} P<0.01$ versus sham control.

U0126 elicited a significant decrease in Egr-1 expression and myocardial $\mathrm{I} / \mathrm{R}$ injury. These data indicated that the cardiac protective effect of EA on myocardial I/R may be partially mediated by the attenuation of ERK1/2 activation and decrease in the downstream Egr-lexpression in the myocardium.

Egr-1 is initially linked to the control of cell growth, survival, and transformation [24]. Recently, it has been implicated as a "master switch" in the injury response in a variety of models including vascular restenosis as well as I/R injury of many organs such as lung, heart, gut, and kidney $[14,25-28]$. However, the upstream components capable of activating Egr-1 in myocardial I/R is still unclear. In a mouse lung I/R model, the ERK1/2 pathway was demonstrated to trigger Egr-1 expression and subsequent inflammatory damage [14]. In the current study, the change in Egr-1 after I/R parallels that of p-ERK1/2 at both mRNA and protein levels. After blocking the activation of ERK1/2 with U0126, the Egr1 expression was significantly reduced and the myocardial injury was attenuated as well. Thus, our findings suggest that 


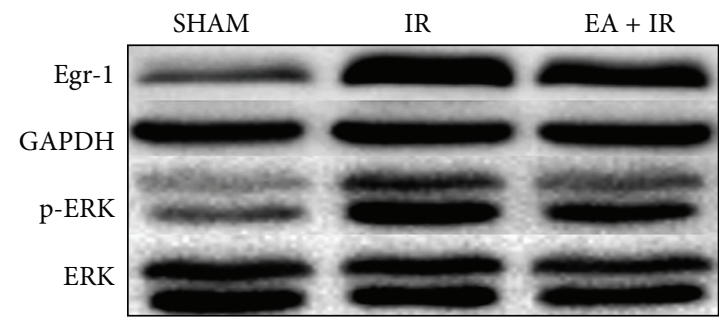

(a)
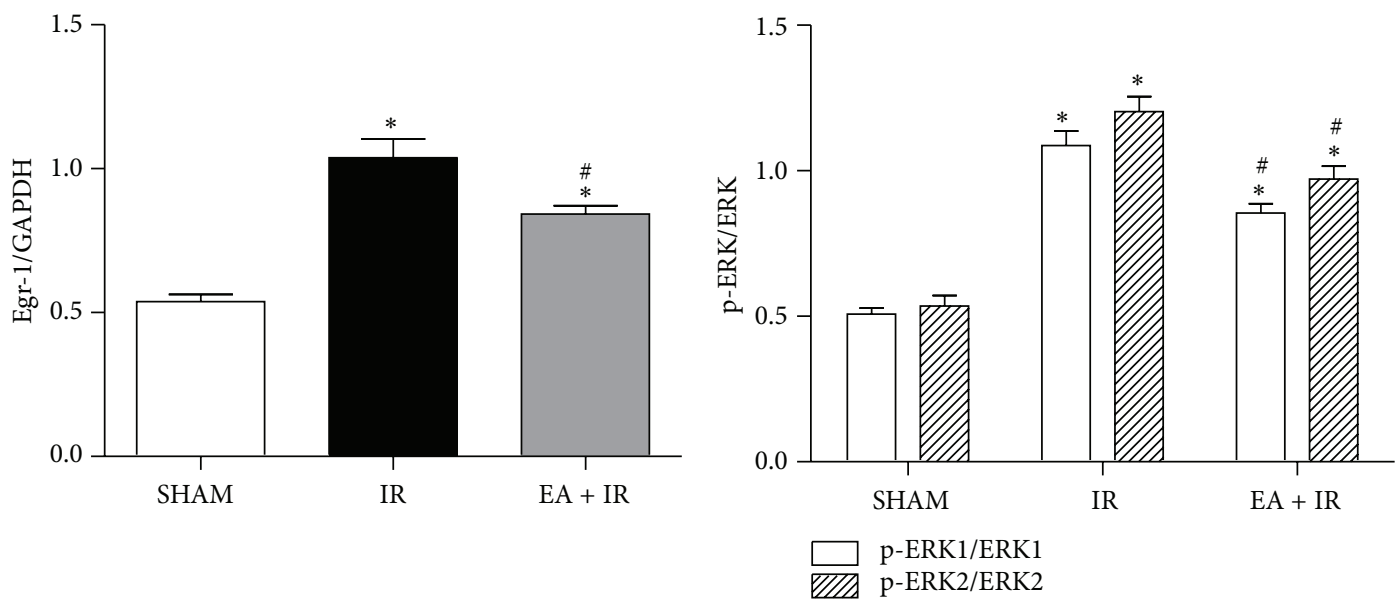

(b)

(c)

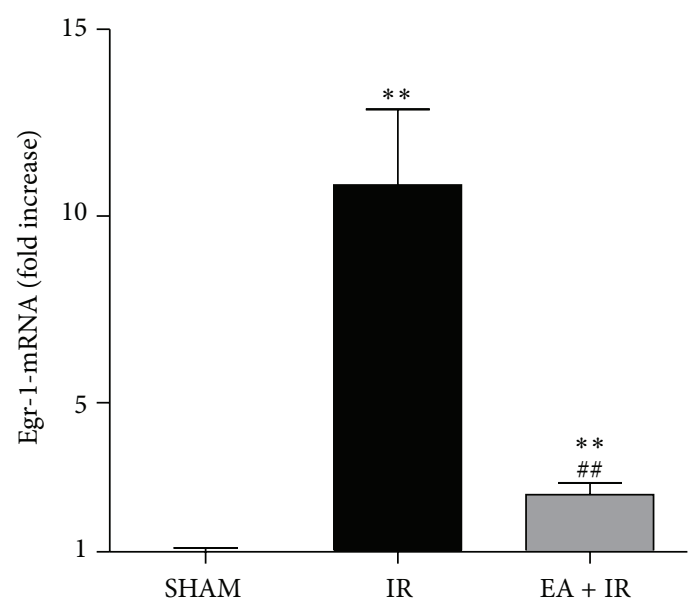

(d)

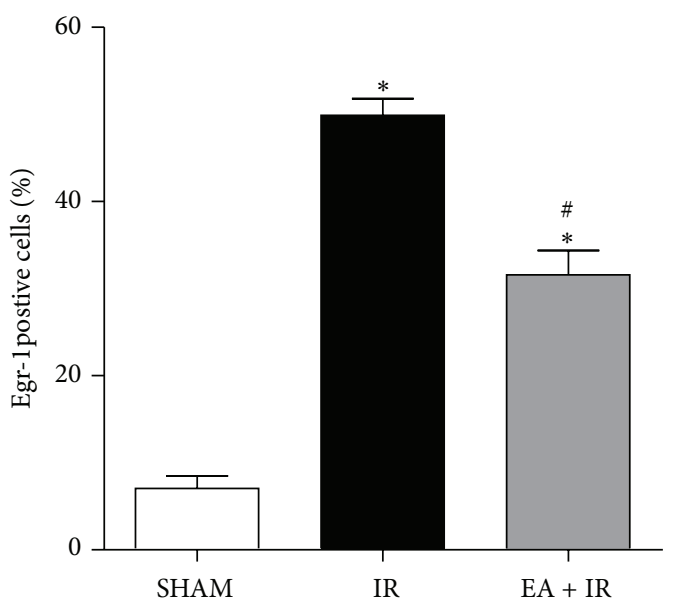

(e)

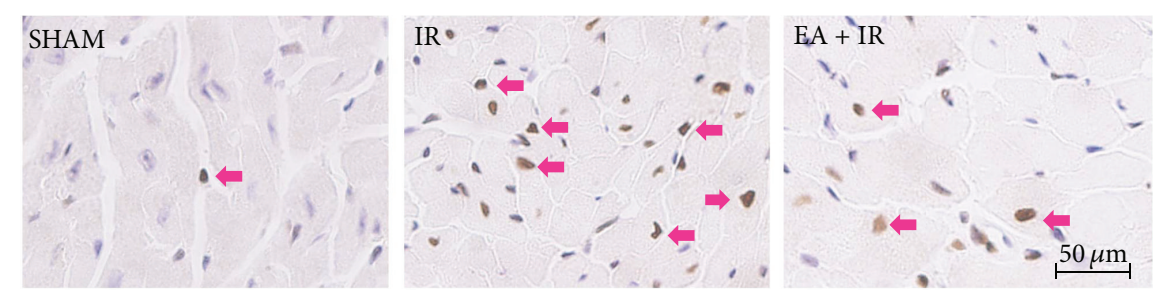

(f)

FIGURE 2: EA inhibited Egr-1 expression and ERK1/2 activation in myocardium undergoing myocardial I/R. Mice were divided into 3 groups: SHAM, IR (myocardial I/R), and EA + IR (EA stimulation was performed $30 \mathrm{~min}$ before myocardial I/R surgery and lasted for 30 min). After $3 \mathrm{~h}$ of reperfusion, the animals were sacrificed and the protein levels of Egr-land p-ERK1/2 were measured by western blot (a) and densitometric analysis is shown in panel (b-c) $(n=3$ /group). The mRNA levels of Egr-1 in these three groups are represented as the relative fold increase versus sham controls ( $\mathrm{d}, n=3$ /group). Immunohistochemical staining of Egr-1 was performed and the quantitation results of Egr-1positive cells were shown in panel (e). Representative images were shown in panel (f). Pink arrows indicate Egr-1positive cells. Scale bar $=50 \mu \mathrm{m} .{ }^{*} P<0.05$ versus sham control; ${ }^{\#} P<0.05$ versus IR; ${ }^{* *} P<0.01$ versus sham control; ${ }^{\# \#} P<0.01$ versus IR. 


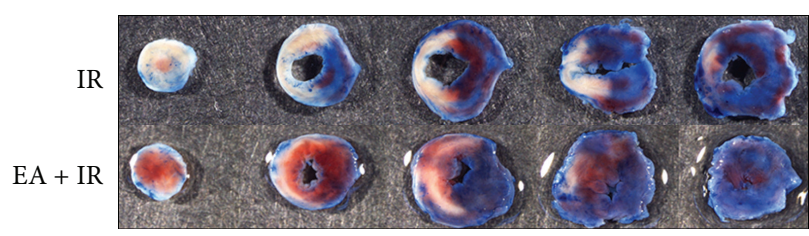

(a)

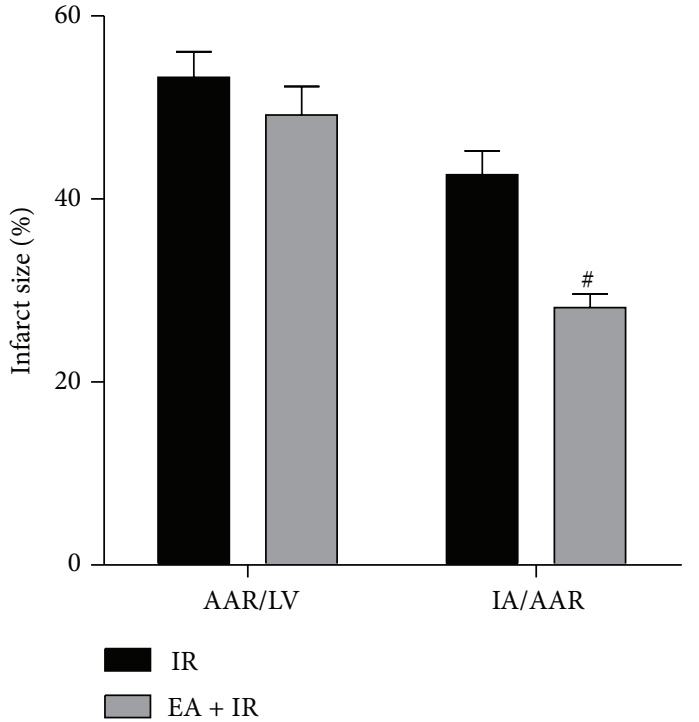

(b)

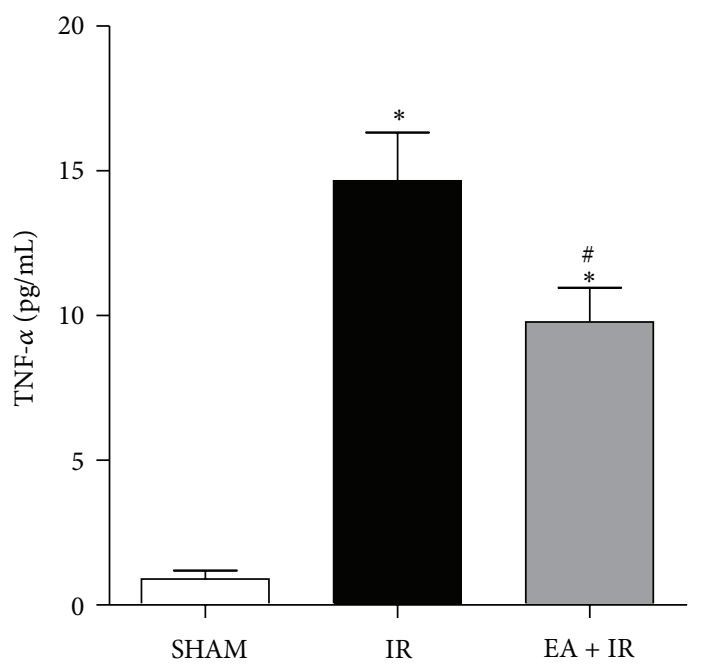

(d)

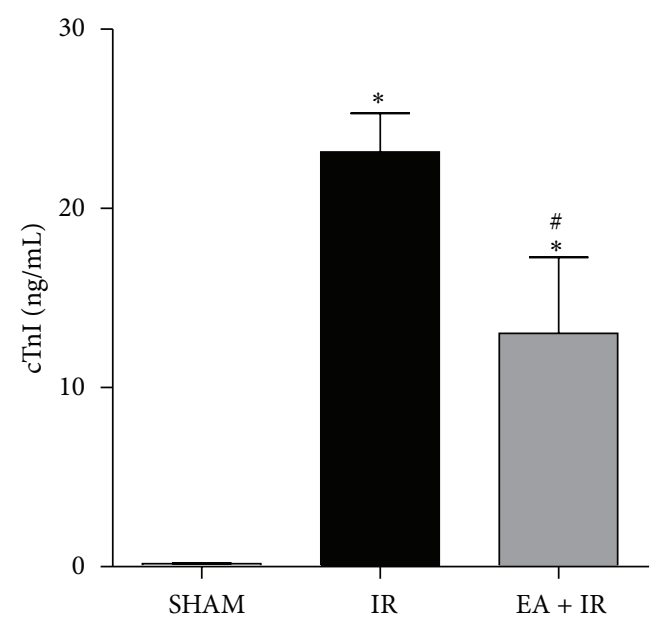

(c)

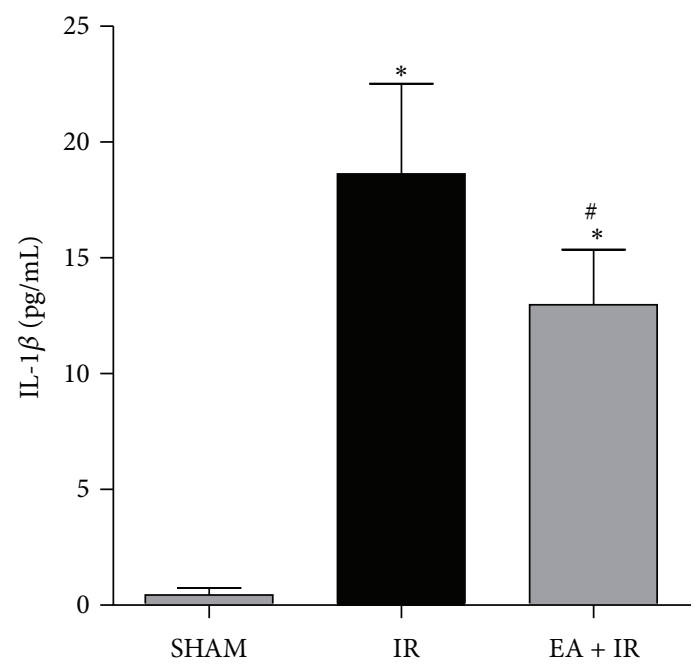

(e)

Figure 3: EA attenuated I/R-induced myocardial tissue damage and inflammatory response. After $24 \mathrm{~h}$ of reperfusion, Evans blue/TTC staining was applied to measure the infarct size ( (a-b) $n=6 /$ group). AAR/LV: area at risk/left ventricle area; IA/AAR: infarct area/area at risk. After $3 \mathrm{~h}$ of reperfusion, the serum cTnI level (c, $n=6 /$ group) and the myocardial levels of TNF- $\alpha$ and IL-1 $\beta$ ((d-e), $n=3 /$ group) were determined using ELISA. ${ }^{*} P<0.01$ versus sham control; ${ }^{*} P<0.05$ versus IR alone.

ERK1/2 is an important upstream signal for modulating Egr-1 expression underlying myocardial ischemia stress.

Although ERK1/2 has been generally reported to be an important member of prosurvival kinases in ischemic preconditioning [29], its role in myocardial ischemia stress remains controversial. In both $\mathrm{I} / \mathrm{R}$-injured rat hearts and hypoxia-reoxygenation-injured cardiomyocytes, inhibiting
ERK1/2 activation reverses the reactive oxygen species production and intracellular $\mathrm{Ca}^{2+}$ overload [30]. Furthermore, treating hypertrophic $\mathrm{H} 9 \mathrm{c} 2$ cells with $\mathrm{U} 0126$ reduces the DNA fragmentation and nuclear condensation [31]. These findings are consistent with our data that blocking the activation of ERK1/2 with U0126 alleviates myocardial I/R injury. 


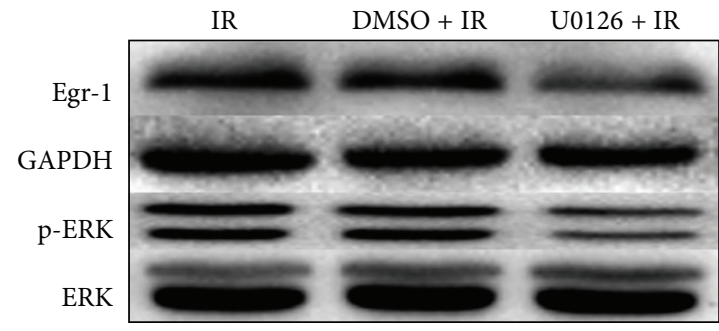

(a)

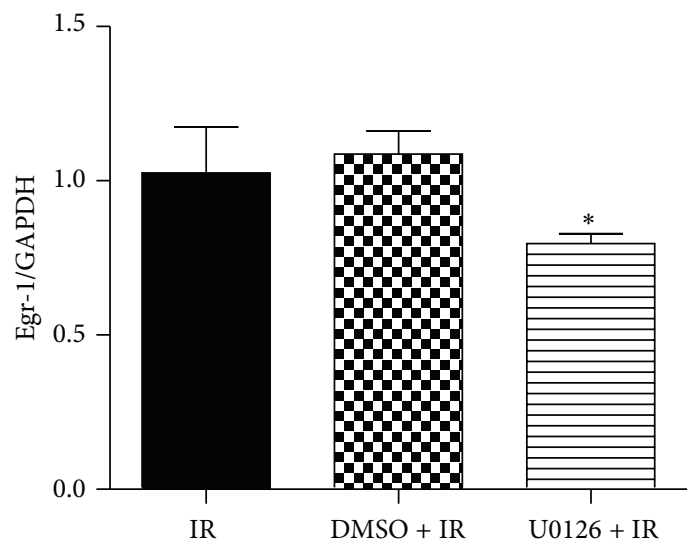

(b)

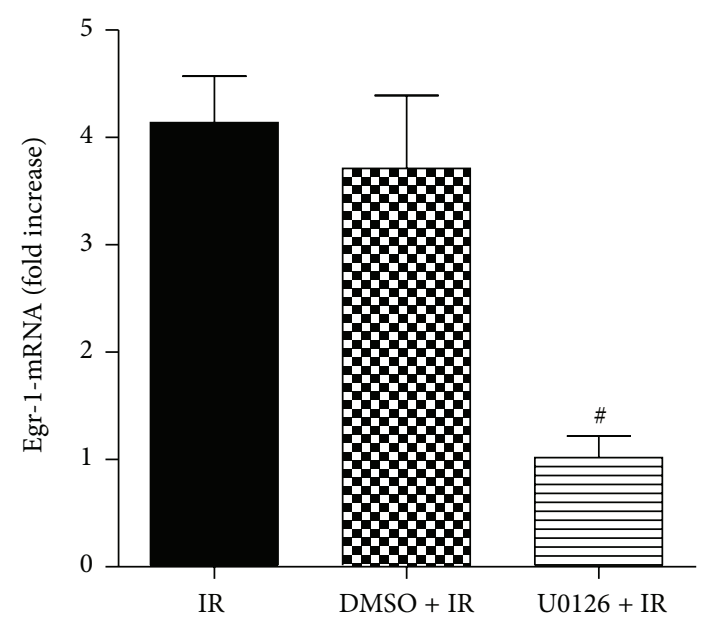

(d)

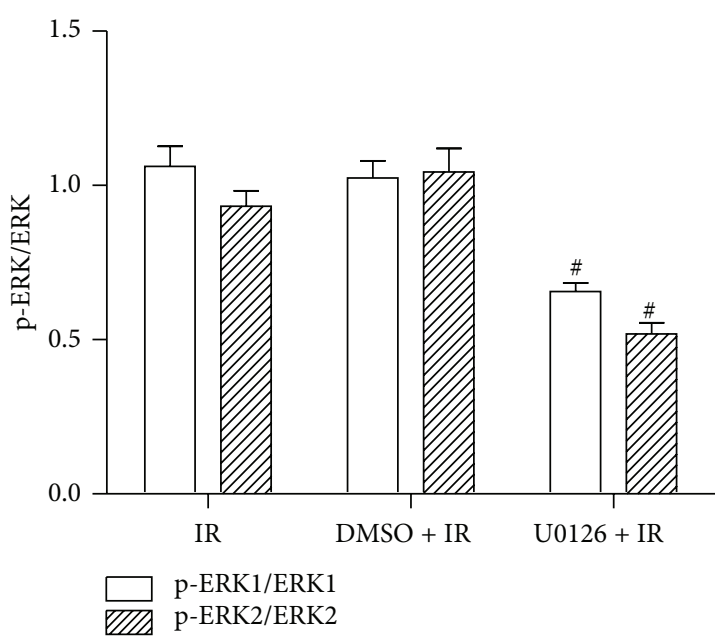

(c)

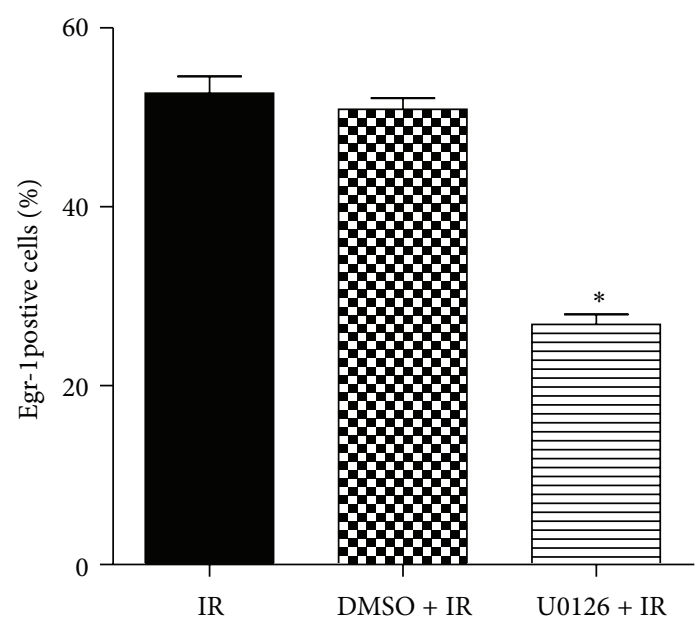

(e)

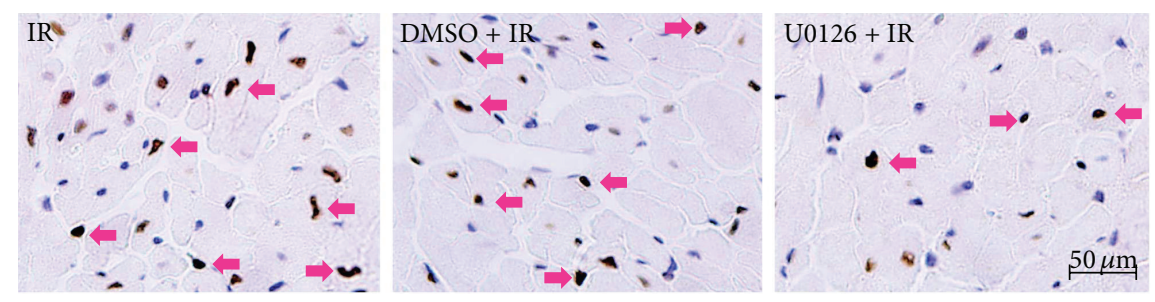

(f)

FIGURE 4: ERK1/2 activation is responsible for Egr-lexpression during myocardial I/R injury. Mice received U0126 (an inhibitor of ERK1/2 kinase, $20 \mathrm{mg} / \mathrm{kg}$, i.p.) or its vehicle $0.1 \% \mathrm{v} / \mathrm{v}$ DMSO treatment before surgery. As described previously, myocardial expression of Egr-1 and p-ERK1/2 was measured using western blot (a). The corresponding densitometric analysis is shown in (b-c) $(n=3 /$ group). The mRNA levels of Egr-1 are shown as fold increase versus U0126 + IR ( $\mathrm{d}, n=3$ /group). Immunohistochemical staining of Egr-1 was performed and the quantitation results of Egr-1positive cells were shown in panel (e). Representative images were shown in panel (f) ( 3 mice /group). Pink arrows indicate Egr-1positive cells. Scale bar $=50 \mu \mathrm{m} .{ }^{*} P<0.05$ and ${ }^{\#} P<0.01$ versus DMSO + IR control. 


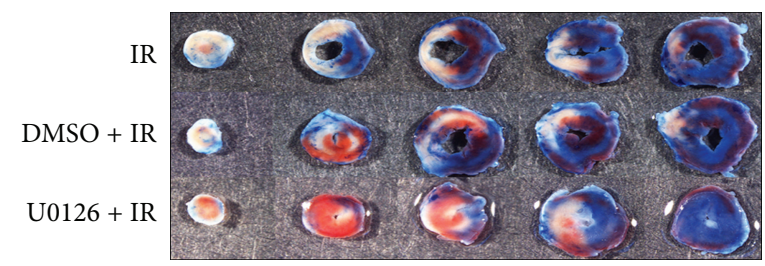

(a)
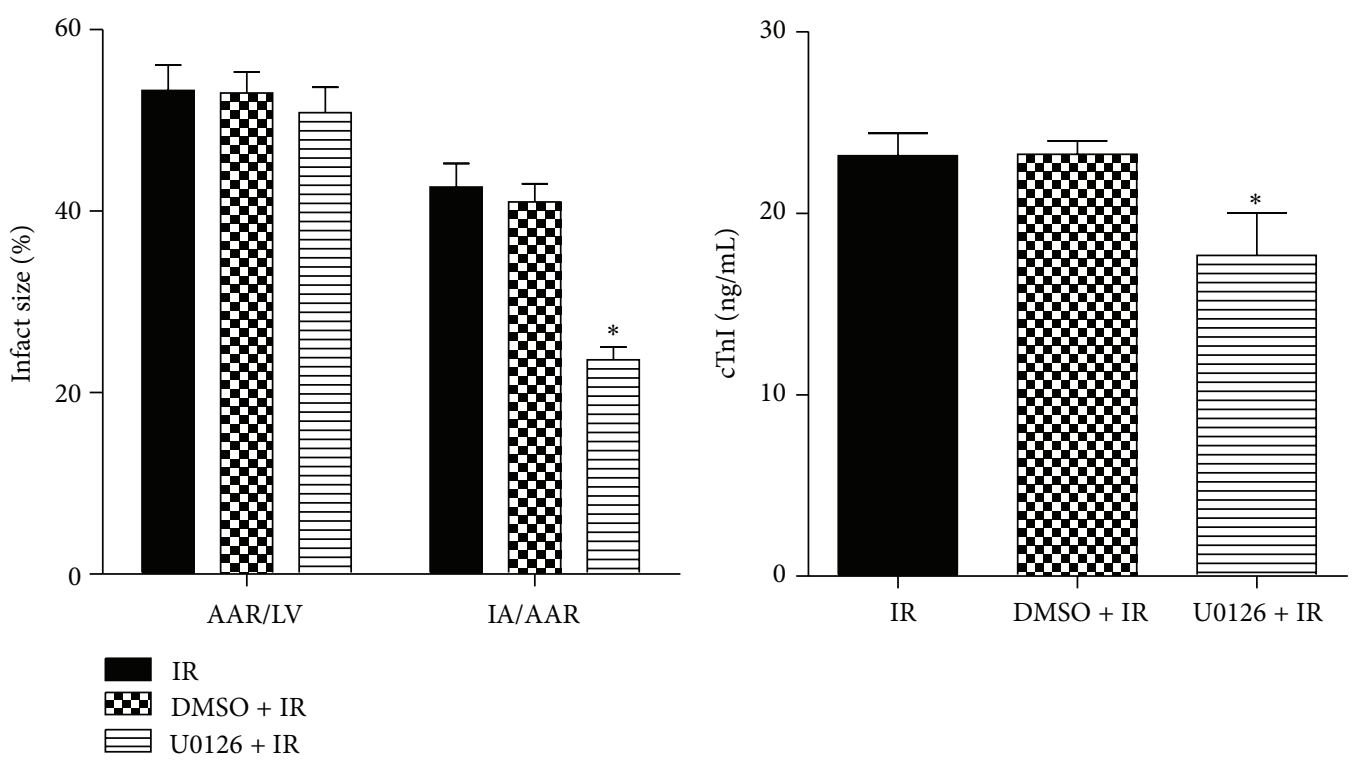

(b)

(c)

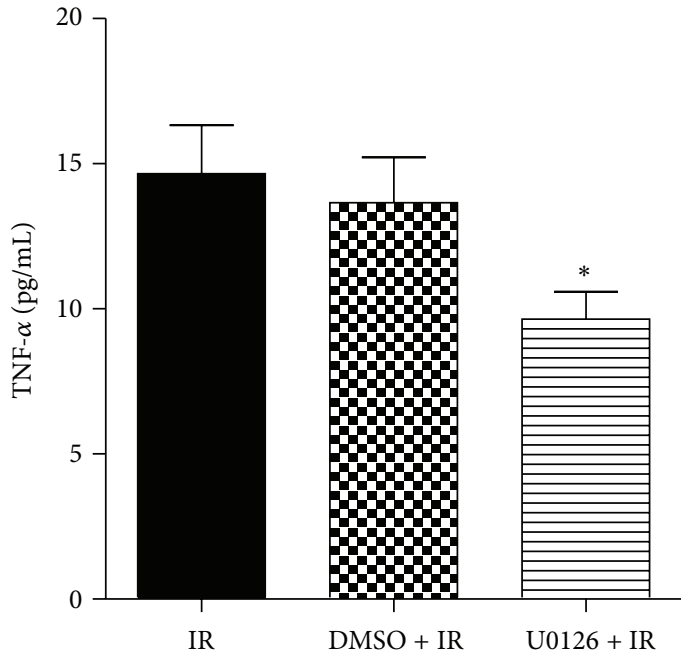

(d)

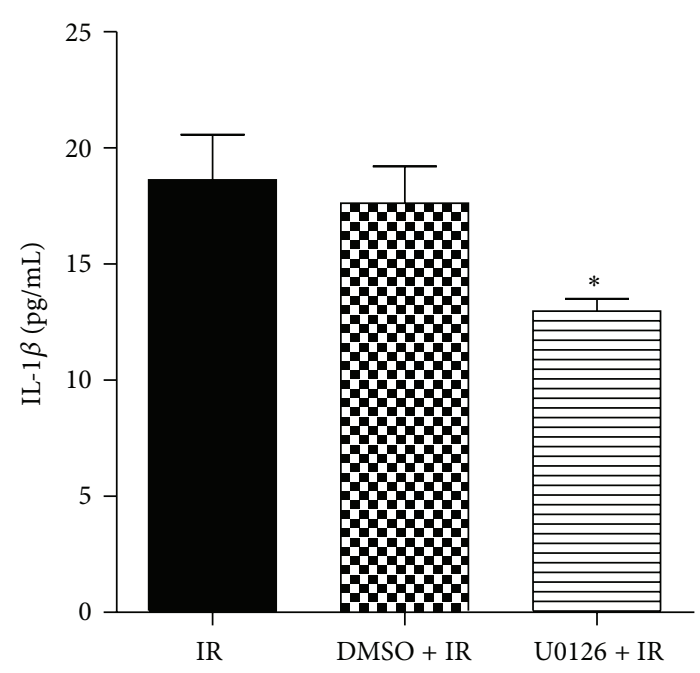

(e)

FIGURE 5: Inhibiting ERK1/2 activation with U0126 protected the myocardium against I/R injury. After 24 h of reperfusion, Evans blue/TTC staining was applied to measure the infarct size ((a-b) $n=6 /$ group). AAR/LV: area at risk/left ventricle area; IA/AAR: infarct area/area at risk. After $3 \mathrm{~h}$ of reperfusion, the serum cTnI level (c, $n=6$ /group) and the myocardial levels of TNF- $\alpha$ and IL-1 $\beta$ ((d-e) $n=3 /$ group) were determined using ELISA. ${ }^{*} P<0.05$ versus DMSO + IR.

EA can regulate the activation of ERK1/2 to produce antiinflammatory [32] and analgesic effects [33, 34]. In a middle cerebral artery occlusion (MCAO) model, EA at either GV20 (Baihui) or DU26 (Renzhong) acupoints increases the phosphorylation of ERK1/2 in the ischemic cortex and hippocampus [35]. In contrast, in animal models of both chronic constriction injury (CCI) and cardiac hypertrophy $[16,33]$, EA reduced the expression of p-ERK1/2. Consistently, in the current study, we found the I/R-induced myocardial expression of both $\mathrm{p}$-ERK1/2 and Egr-1 was downregulated by 


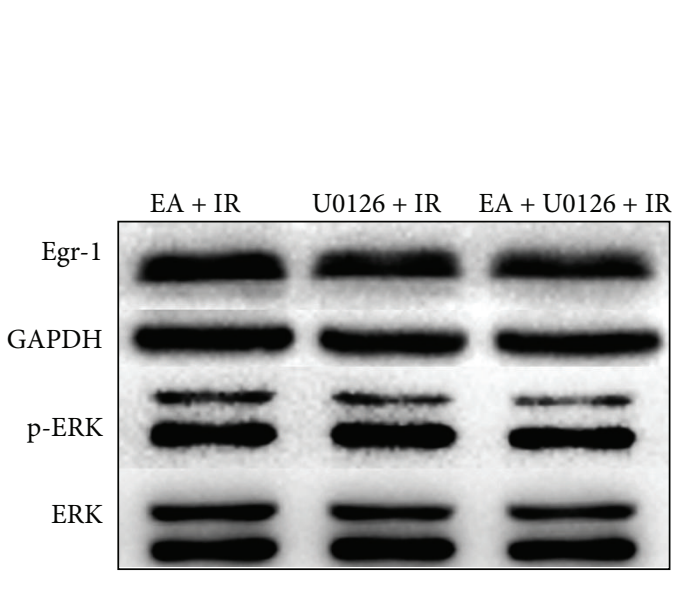

(a)

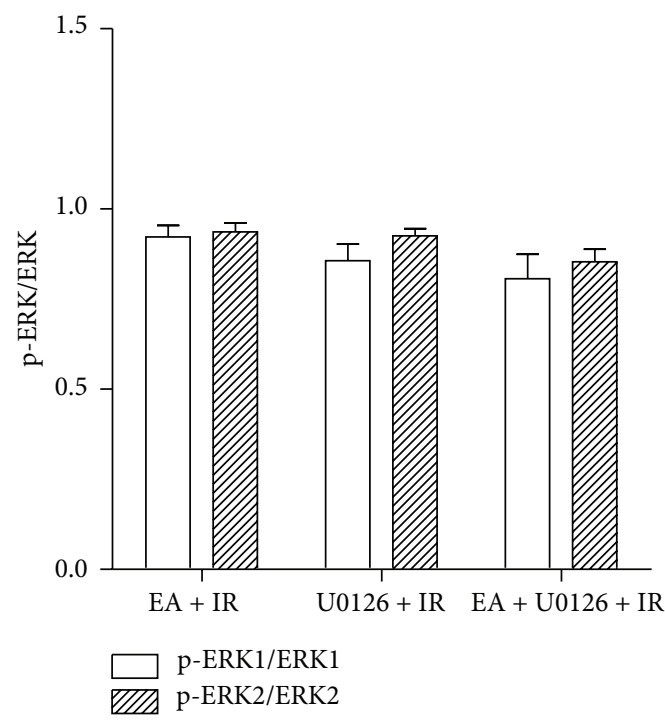

(c)

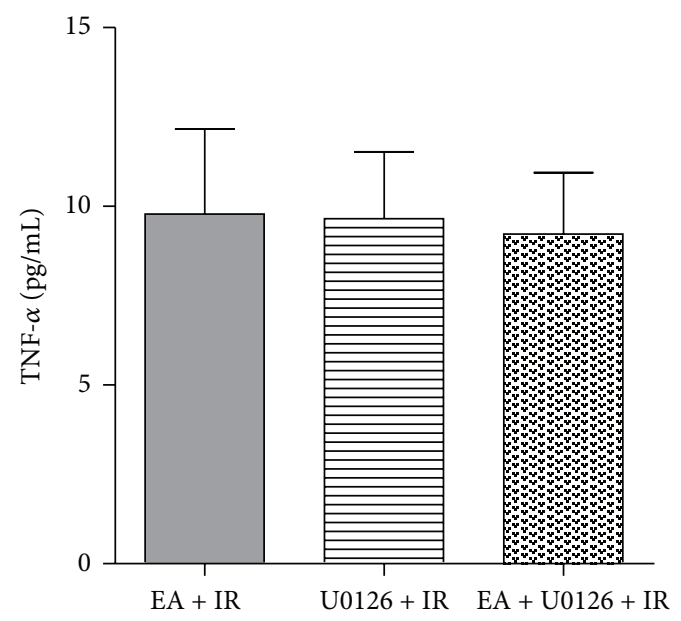

(e)

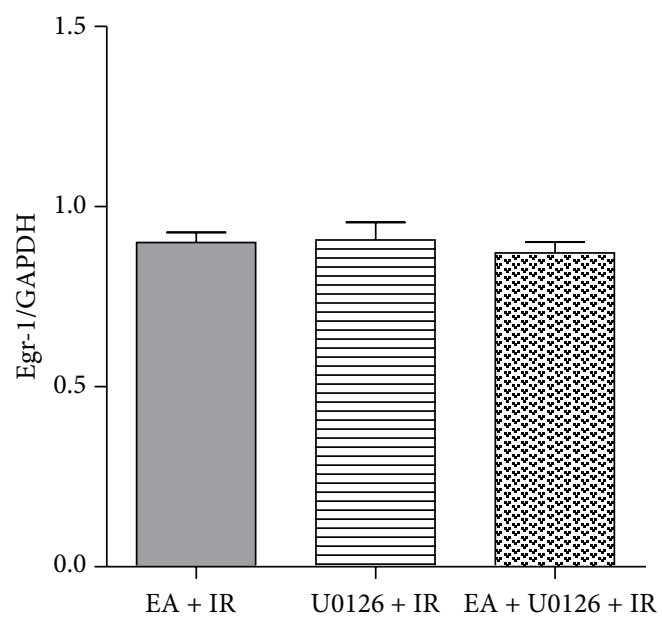

(b)

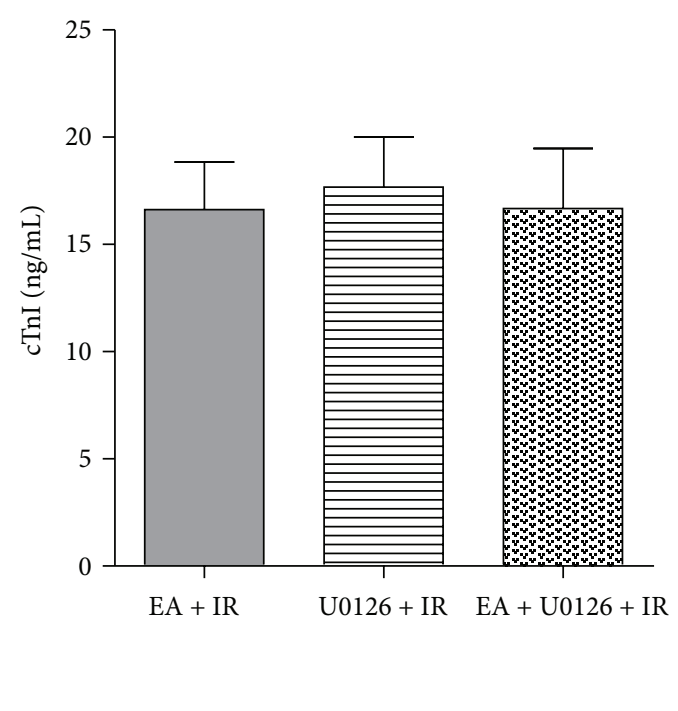

(d)

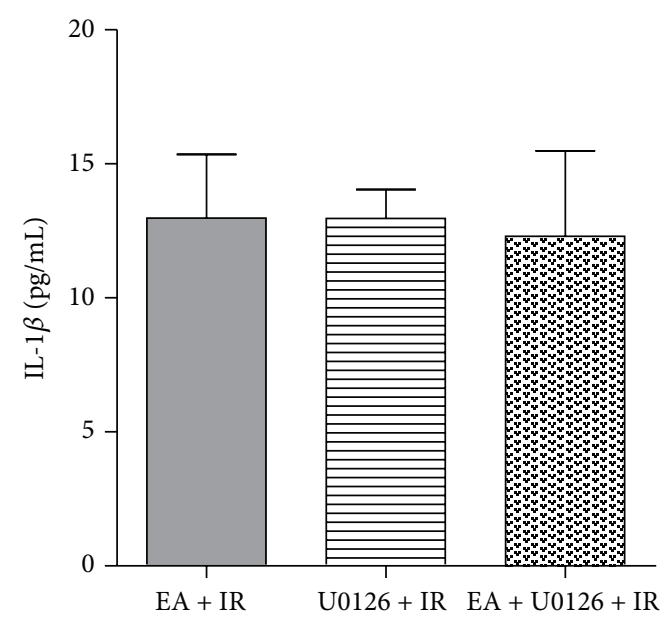

(f)

FIGURE 6: Combination of EA with ERK1/2 inhibitor did not produce more protection against myocardial I/R injury. Combination of EA and U0126 treatment was conducted to investigate the additive effects on ERK1/2/Egr-1 downregulation or the cardiac protective role. Western blot bands and corresponding densitometric analysis of Egr-1 and p-ERK1/2 are shown in $(\mathrm{a}-\mathrm{c})(n=3 /$ group). The myocardial levels of TNF- $\alpha$ and IL-1 $\beta$ ((d-e), $n=3$ /group) as well as the serum level of cTnI (f, $n=6 /$ group) were determined. 
EA at PC6 acupoints. Then we combined EA with the ERK1/2 inhibitor U0126 to investigate if they have an additive effect. However, the combined protocol did not further decrease $\mathrm{p}$ ERK1/2 or Egr-1 expression or protect the myocardium. We speculate that ERK1/2-Egr-1 pathway might be a common target for both EA and U0126, and either EA or U0126 may have already exerted their maximal effects in our present experiment conditions.

In summary, EA treatment at PC6 (Neiguan) acupoints decreased infarct size, reduced the release of proinflammatory cytokines, and inhibited ERK1/2 activation and Egrlexpression in a mouse myocardial I/R injury model. The inhibition of ERK1/2-Egr-1signaling pathway may be, at least in part, responsible for the cardioprotective effects of EA against I/R injury.

\section{Conflict of Interests}

The authors declared no conflict of interests.

\section{Authors' Contribution}

Juan Zhang and Jiangang Song contributed equally.

\section{Acknowledgments}

This research was supported by the National Key Basic Research Program (973 Program) of China (Grant no. 2013CB531900, Beijing, China) and National Natural Science Foundation of China (Grant nos. 81001545, 81272142, Beijing, China).

\section{References}

[1] F. J. Zijlstra, I. V. D. Berg-de Lange, F. J. P. M. Huygen, and J. Klein, "Anti-inflammatory actions of acupuncture," Mediators of Inflammation, vol. 12, no. 2, pp. 59-69, 2003.

[2] L. Yang, J. Yang, Q. Wang et al., "Cardioprotective effects of electroacupuncture pretreatment on patients undergoing heart valve replacement surgery: a randomized controlled trial," Annals of Thoracic Surgery, vol. 89, no. 3, pp. 781-786, 2010.

[3] M.-T. Tsou, C.-H. Huang, and J.-H. Chiu, "Electroacupuncture on PC6 (Neiguan) attenuates ischemia/reperfusion injury in rat hearts," American Journal of Chinese Medicine, vol. 32, no. 6, pp. 951-965, 2004.

[4] H. L. Lujan, V. J. Kramer, and S. E. DiCarlo, "Electroacupuncture decreases the susceptibility to ventricular tachycardia in conscious rats by reducing cardiac metabolic demand," American Journal of Physiology: Heart and Circulatory Physiology, vol. 292, no. 5, pp. H2550-H2555, 2007.

[5] J. C. Longhurst, "Electroacupuncture treatment of arrhythmias in myocardial ischemia," American Journal of Physiology: Heart and Circulatory Physiology, vol. 292, no. 5, pp. H2032-H2034, 2007.

[6] J. Zhang, X. H. Jia, Z. W. Xu et al., "Improved mesenchymal stem cell survival in ischemic heart through electroacupuncture," Chinese Journal of Integrative Medicine, vol. 19, no. 8, pp. 573581, 2012.

[7] W. Zhou, Y. Ko, P. Benharash et al., "Cardioprotection of electroacupuncture against myocardial ischemia-reperfusion injury by modulation of cardiac norepinephrine release," American Journal of Physiology: Heart and Circulatory Physiology, vol. 302, no. 9, pp. H1818-H1825, 2012.

[8] J. Gao, W. Fu, Z. Jin, and X. Yu, "A preliminary study on the cardioprotection of acupuncture pretreatment in rats with ischemia and reperfusion: involvement of cardiac $\beta$ adrenoceptors," Journal of Physiological Sciences, vol. 56, no. 4, pp. 275-279, 2006.

[9] X. Ni, Y. Xie, Q. Wang et al., "Cardioprotective effect of transcutaneous electric acupoint stimulation in the pediatric cardiac patients: a randomized controlled clinical trial," Paediatric Anaesthesia, vol. 22, no. 8, pp. 805-811, 2012.

[10] L. M. Khachigian, "Early growth response-1 in cardiovascular pathobiology," Circulation Research, vol. 98, no. 2, pp. 186-191, 2006.

[11] Y. Zhang, G. Shi, J. Zheng et al., "The protective effect of Egr-1 antisense oligodeoxyribonucleotide on myocardial injury induced by ischemia-reperfusion and hypoxia-reoxygenation," Cellular Physiology and Biochemistry, vol. 22, no. 5-6, pp. 645652, 2008.

[12] S.-F. Yan, T. Fujita, J. Lu et al., "Egr-1, a master switch coordinating upregulation of divergent gene families underlying ischemic stress," Nature Medicine, vol. 6, no. 12, pp. 1355-1361, 2000.

[13] R. Bhindi, R. G. Fahmy, A. C. Mcmahon, L. M. Khachigian, and H. C. Lowe, "Intracoronary delivery of DNAzymes targeting human EGR-1 reduces infarct size following myocardial ischaemia reperfusion," The Journal of Pathology, vol. 227, no. 2, pp. 157-164, 2012.

[14] T. Fujita, T. Asai, M. Andrassy et al., "PKC $\beta$ regulates ischemia/reperfusion injury in the lung," The Journal of Clinical Investigation, vol. 113, no. 11, pp. 1615-1623, 2004.

[15] T. Hartney, R. Birari, S. Venkataraman et al., "Xanthine oxidasederived ROS upregulate Egr-1 via ERK1/2 in PA smooth muscle cells; model to test impact of extracellular ROS in chronic hypoxia," PLoS ONE, vol. 6, no. 11, Article ID e27531, 2011.

[16] H. Wang, J. Li, J. Li et al., "Electroacupuncture at PC6 (Neiguan) improves extracellular signal-regulated kinase signaling pathways through the regulation of neuroendocrine cytokines in myocardial hypertrophic rats," Evidence-Based Complementary and Alternative Medicine, vol. 2012, Article ID 792820, 9 pages, 2012.

[17] Y. Toyoda, S. Endo, K. Tsuneyama et al., "Mechanism of exacerbative effect of progesterone on drug-induced liver injury," Toxicological Sciences, vol. 126, no. 1, pp. 16-27, 2012.

[18] S. Göser, M. Andrassy, S. J. Buss et al., "Cardiac troponin I but not cardiac troponin $\mathrm{T}$ induces severe autoimmune inflammation in the myocardium," Circulation, vol. 114, no. 16, pp. 1693-1702, 2006.

[19] J. G. dos Santos Jr., R. Filev, C. T. Coelho, Y. Yamamura, L. E. Mello, and A. Tabosa, "Electroacupuncture inhibits ethanolinduced locomotor sensitization and alters homerlA mRNA expression in Mice," Alcoholism: Clinical and Experimental Research, vol. 33, no. 8, pp. 1469-1475, 2009.

[20] J.-G. Song, H.-H. Li, Y.-F. Cao et al., "Electroacupuncture improves survival in rats with lethal endotoxemia via the autonomic nervous system," Anesthesiology, vol. 116, no. 2, pp. 406-414, 2012.

[21] M. Kawaguchi, M. Takahashi, T. Hata et al., "Inflammasome activation of cardiac fibroblasts is essential for myocardial ischemia/reperfusion injury," Circulation, vol. 123, no. 6, pp. 594-604, 2011. 
[22] X.-R. Wang, H. Lin, and Z.-H. Wang, "Protective effects of electroacupuncture and Salviae miltiorrhizae on myocardial ischemia/reperfusion in rabbits," Acupuncture and ElectroTherapeutics Research, vol. 28, no. 3-4, pp. 175-182, 2003.

[23] X.-R. Wang, J. Xiao, and D.-J. Sun, "Myocardial protective effects of electroacupuncture and hypothermia on porcine heart after ischemia/reperfusion," Acupuncture and ElectroTherapeutics Research, vol. 28, no. 3-4, pp. 193-200, 2003.

[24] G. Thiel and G. Cibelli, "Regulation of life and death by the zinc finger transcription factor Egr-1," Journal of Cellular Physiology, vol. 193, no. 3, pp. 287-292, 2002.

[25] T. Brand, H. S. Sharma, K. E. Fleischmann et al., "Protooncogene expression in porcine myocardium subjected to ischemia and reperfusion," Circulation Research, vol. 71, no. 6, pp. 1351-1360, 1992.

[26] H. Aebert, T. Cornelius, T. Ehr et al., "Expression of immediate early genes after cardioplegic arrest and reperfusion," Annals of Thoracic Surgery, vol. 63, no. 6, pp. 1669-1675, 1997.

[27] Y. Chen, V. C. H. Lui, N. V. Rooijen, and P. K. H. Tam, "Depletion of intestinal resident macrophages prevents ischaemia reperfusion injury in gut," Gut, vol. 53, no. 12, pp. 1772-1780, 2004.

[28] J. V. Bonventre, V. P. Sukhatme, M. Bamberger, A. J. Ouellette, and D. Brown, "Localization of the protein product of the immediate early growth response gene, Egr-1, in the kidney after ischemia and reperfusion," Cell Regulation, vol. 2, no. 3, pp. 251260, 1991.

[29] X. Yang, M. V. Cohen, and J. M. Downey, "Mechanism of cardioprotection by early ischemic preconditioning," Cardiovascular Drugs and Therapy, vol. 24, no. 3, pp. 225-234, 2010.

[30] S.-M. Kang, S. Lim, H. Song et al., "Allopurinol modulates reactive oxygen species generation and $\mathrm{Ca}^{2+}$ overload in ischemiareperfused heart and hypoxia-reoxygenated cardiomyocytes," European Journal of Pharmacology, vol. 535, no. 1-3, pp. 212-219, 2006.

[31] S.-D. Lee, S.-H. Chang, W.-H. Kuo et al., "Role of mitogenactivated protein kinase kinase in Porphyromonas gingivalisinduced myocardial cell hypertrophy and apoptosis," European Journal of Oral Sciences, vol. 114, no. 2, pp. 154-159, 2006.

[32] J. Du, Q. Wang, B. Hu et al., "Involvement of ERK 1/2 activation in electroacupuncture pretreatment via cannabinoid CB1 receptor in rats," Brain Research, vol. 1360, pp. 1-7, 2010.

[33] J. Yu, C. Zhao, and X. Luo, "The effects of electroacupuncture on the extracellular signal-regulated kinase $1 / 2 / \mathrm{P} 2 \mathrm{X} 3$ signal pathway in the spinal cord of rats with chronic constriction injury," Anesthesia \& Analgesia, vol. 116, no. 1, pp. 239-246, 2012.

[34] D. C. Choi, J. Y. Lee, E. J. Lim, H. H. Baik, T. H. Oh, and T. Y. Yune, "Inhibition of ROS-induced p38MAPK and ERK activation in microglia by acupuncture relieves neuropathic pain after spinal cord injury in rats," Experimental Neurology, vol. 236, no. 2, pp. 268-282, 2012.

[35] L. Zhao, Y. Wang, N. Sun, X. Liu, L. Li, and J. Shi, "Electroacupuncture regulates TRPM7 expression through the trkA/PI3K pathway after cerebral ischemia-reperfusion in rats," Life Sciences, vol. 81, no. 15, pp. 1211-1222, 2007. 


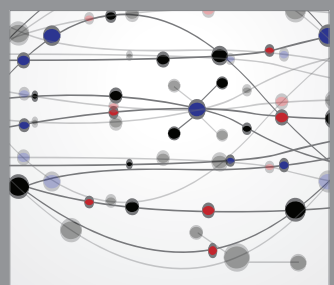

The Scientific World Journal
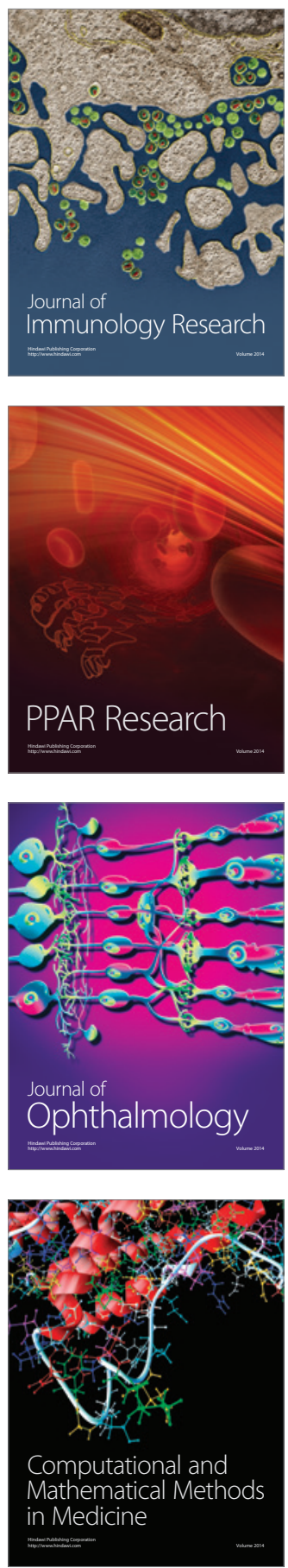

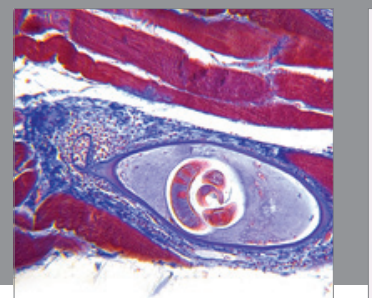

Gastroenterology

Research and Practice
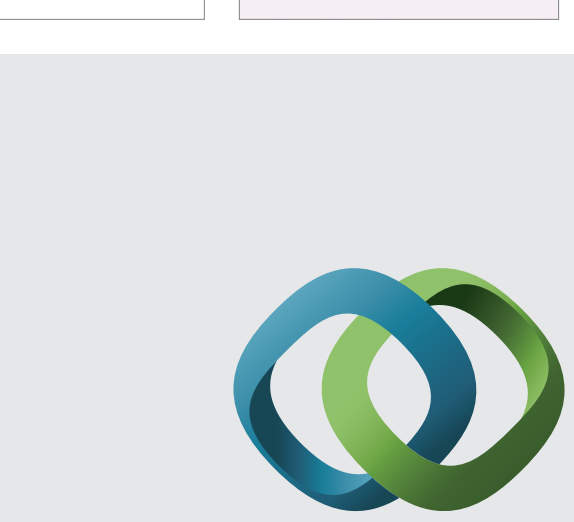

\section{Hindawi}

Submit your manuscripts at

http://www.hindawi.com
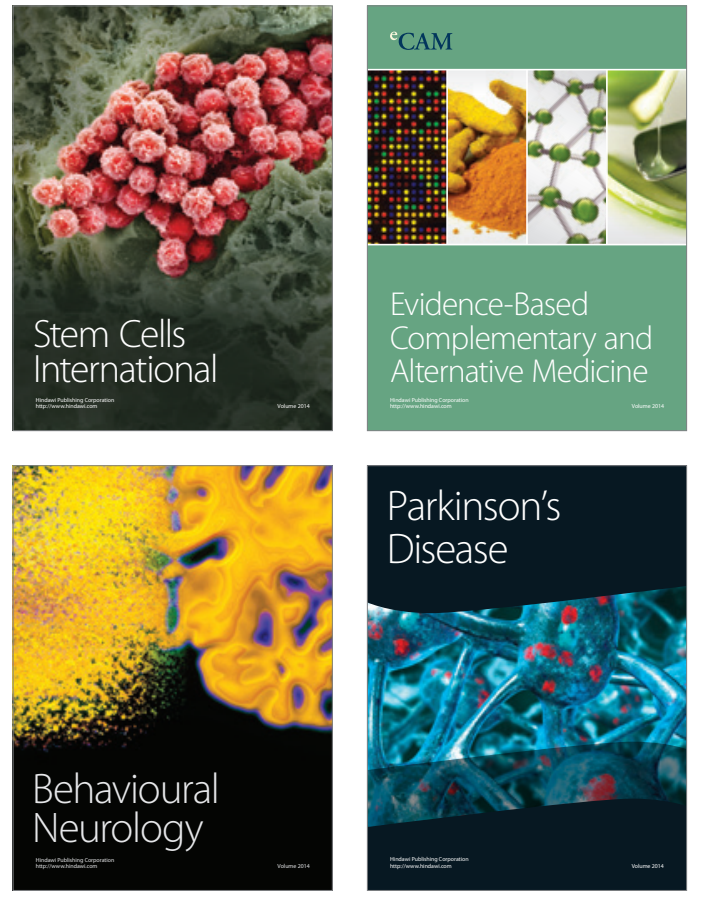
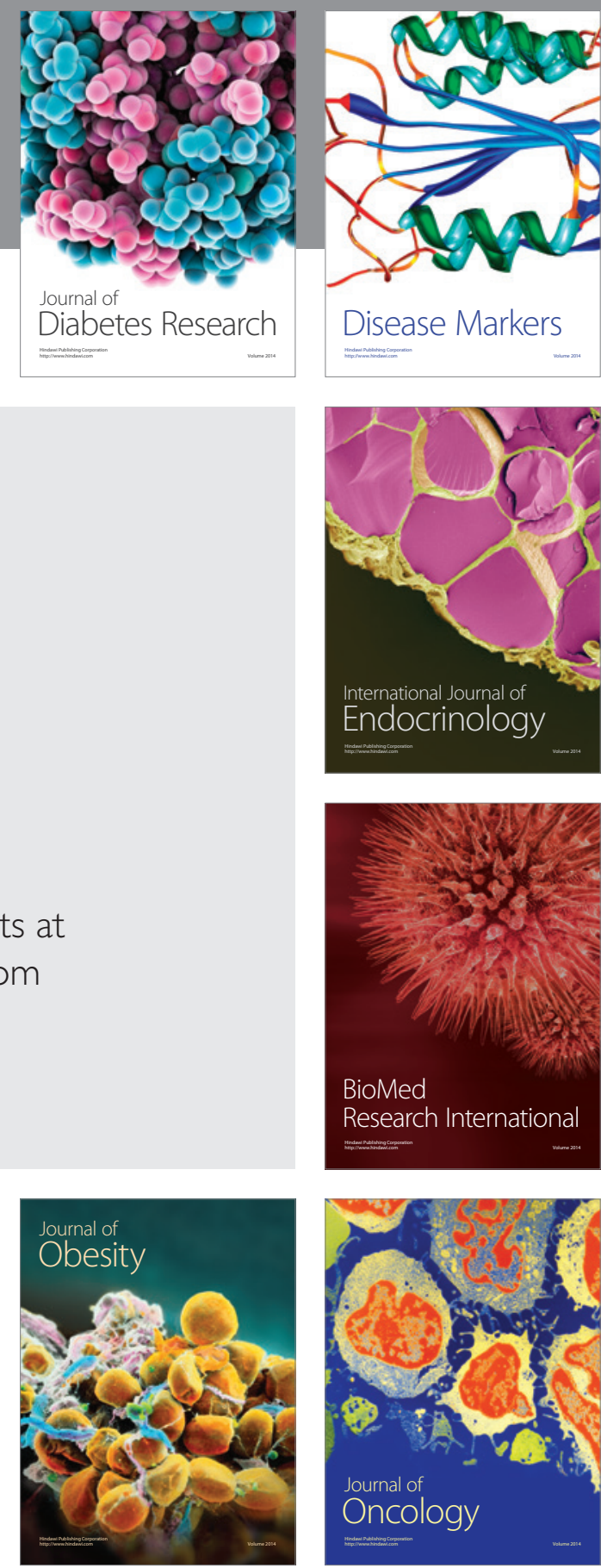

Disease Markers
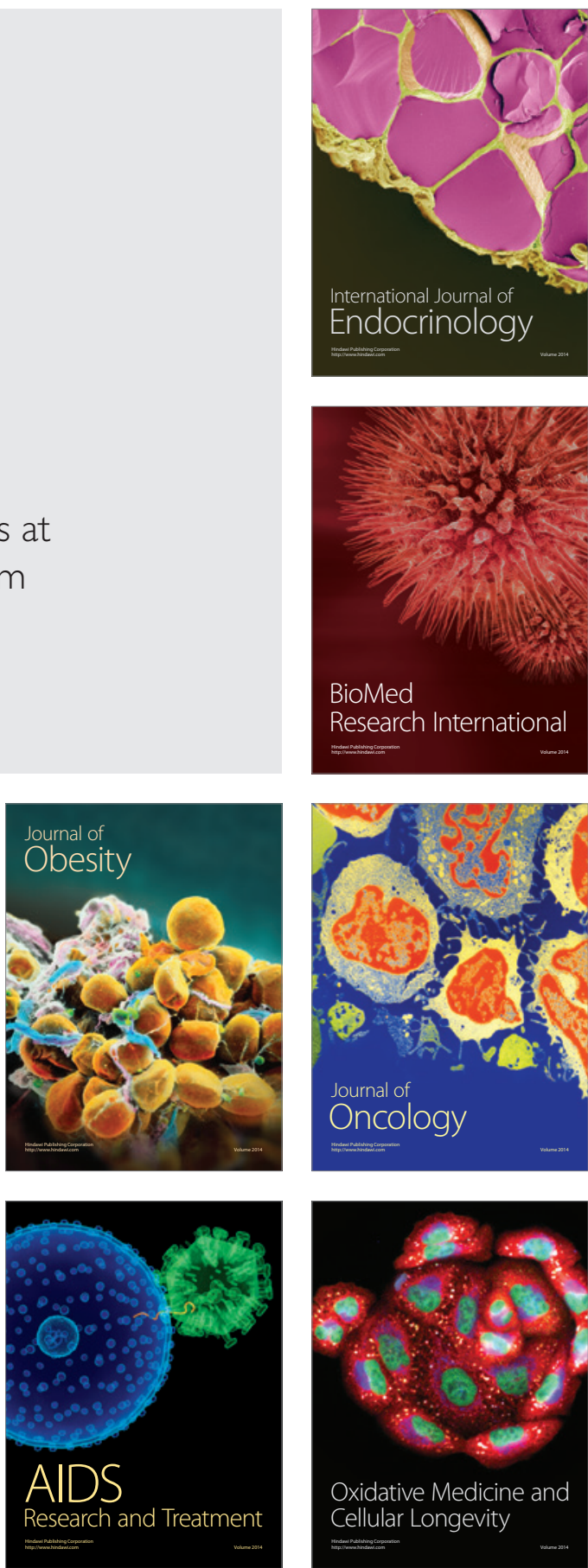